\title{
Neue Leistungspolitik bei Angestellten - Impulse durch ERA?
}

\begin{abstract}
Auf den ständig steigenden Leistungsdruck in den Angestelltenbereichen haben Betriebsräte und Gewerkschaft bislang kaum eine Antwort. Einen Ansatzpunkt könnte die Regulierung von Leistungsentgelten im Angestelltenbereich darstellen. Die Entgeltrahmenabkommen (ERA-Tarifverträge) in der Metall- und Elektroindustrie bieten dazu neue Möglichkeiten. Ob und wie Leistungsentgelt tatsächlich auch als ein leistungspolitisches Instrument genutzt wird oder genutzt werden könnte, hängt aber von einer anderen Frage ab: Wie funktioniert überhaupt Leistungssteuerung bei Angestellten?
\end{abstract}

\section{Mit Leistungsentgelt Leistung regulieren?}

Galt über lange Jahre die Arbeitssituation von Angestellten als privilegiert und galten die ihnen zugestandenen Freiräume als Ausdruck dieser Privilegierung, mehren sich in den letzten Jahren die Beobachtungen eines zunehmenden Arbeits- und Leistungsdrucks, von überbordenden Arbeitszeiten und Überlastung (Grewer et al. 2007; Kotthoff/Wagner 2008). Anders als beim Leistungslohn in der Produktion haben Betriebsräte und Gewerkschaften aber bei den Arbeits- und Leistungsbedingungen im Zeitlohn- bzw. Angestelltenbereich bislang kaum etwas mitzureden. Mit dem Abschluss des neuen Entgeltrahmenabkommens (ERA) in der deutschen Metall -und Elektroindustrie verfolgte die IG Metall deshalb nicht nur tarifpolitische, sondern auch leistungs- und angestelltenpolitische Ziele (Huber/Schild 2004).

Die ERA-Tarifverträge enthalten in zweifacher Hinsicht leistungs- (und angestellten)politisches Innovationspotenzial: Zum Ersten ist nun die Übertragung erfolgreicher Ansätze der Leistungsregulierung aus der Produktionsarbeit auf die Angestelltenbereiche zumindest theoretisch möglich. Der Schlüssel dazu ist die jetzt auch im Angestelltenbereichen zulässige Leistungsentlohnung und die damit verbundene Übertragung der starken Mitbestimmung bei der Lohngestaltung auf das - im Angestelltenbereich besonders - schwach regulierte Feld der Leistungspolitik (Schmierl 1995). Neu ist zum Zweiten, dass nun auch Zielvereinbarungen tariflich zulässig und reguliert sind. Folglich ist nicht nur ein neues
Instrument im leistungspolitischen Spiel, sondern auch eine in verschiedener Hinsicht neuartige Methodik (Ehlscheid/Tondorf 2006; Kratzer 2006).

Wer mit einer (Neu-)Gestaltung der Leistungsentgelte im Angestelltenbereich Leistung regulieren und den Leistungsdruck begrenzen will, der muss allerdings erst mal wissen, woher der Leistungsdruck überhaupt kommt, welche Bedeutung Leistungsentgelte bislang haben und - nicht zuletzt - was die beteiligten Akteure davon halten. Wir werden uns deshalb zunächst mit den Ursachen veränderter Leistungsanforderungen und -bedingungen befassen (Abschnitt 2 bis 5) und im Anschluss daran diskutieren, inwiefern Leistungsentgelte ein Instrument einer neuen Leistungspolitik bei Angestellten sein können (Abschnitt 6 und 7).

Wir stützen uns dabei auf die Befunde einer empirischen Untersuchung zu den Chancen und Risiken der ERA-Einführung im Angestelltenbereich, die wir zwischen 2006 und 2008 in insgesamt neun Betrieben der Metall- und Elektroindustrie durchgeführt haben. ${ }^{1}$ In fünf Intensiv- und vier Kurzfallstudien fanden insgesamt gut 70 leitfadengestützte Einzel- und Gruppeninterviews mit rund 180 Personen statt. Interviewt wurden zum einen betriebliche Experten (Betriebsleitung, Management, Betriebsrat und Führungskräfte). Zum anderen haben wir versucht, die Heterogenität des Angestelltenbereichs durch die Differenzierung des Gesamtsamples in vor allem drei „Angestelltengruppen“ einzufangen: Einbezogen wurden hochqualifizierte (hier vor allem: technische) Angestellte, „produktionsnahe Angestellte“ aus Vertrieb, Auftragsbearbeitung, Einkauf und Fertigungssteuerung (mit zumeist mittleren kaufmännischen oder technischen Qualifikationen) sowie Teamassistentinnen (in der Regel Bürokauffrauen).

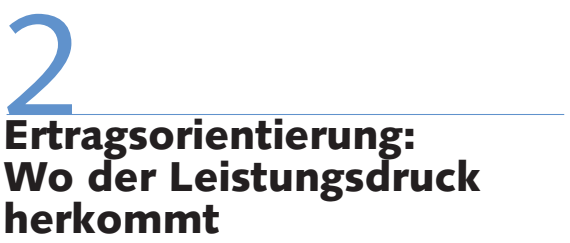

Der Leistungsdruck, mit dem die Angestellten in zunehmendem Maße konfrontiert sind, ist das Ergebnis von zwei miteinander verknüpften neuen Steuerungsprinzipien: Erstens führt die Ausrichtung an abstrakten und dynamischen Ergebnisvorgaben zu einer systematischen Überlastung der Organisation. Und zweitens wird das daraus resultierende organisationelle

\footnotetext{
1 Die Untersuchung wurde im Rahmen des Projekts "Leistungsgestaltung im Angestelltenbereich Chancen und Risiken der ERA-Einführung "vom ISF München (Nick Kratzer, Sarah Nies) durchgeführt. Dieses Projekt wurde von der Hans-BöcklerStiftung von November 2005 bis Juni 2008 gefördert. Ausführlich werden die Befunde dargestellt in: Kratzer/Nies (2009). Wir bedanken uns bei zwei anonymen Gutachtern/Gutachterinnen der WSIMitteilungen für ihre Anmerkungen zu diesem Beitrag
}

Nick Kratzer, Dr., Mitarbeiter am Institut für Sozialwissenschaftliche Forschung (ISF) München. Arbeitsschwerpunkte: Leistungspolitik, Subjektivierung von Arbeit, Entgrenzung von Arbeit und Leben. e-mail: nick.kratzer@isf-muenchen.de Sarah Nies, Dipl.-Soz., Mitarbeiterin am ISF München. Arbeitsschwerpunkte: Leistungspolitik, Arbeit und Interesse. e-mail: sarah.nies@isf-muenchen.de 
Problem in ein individuelles Problem transformiert: Die Bewältigung der „systematischen Überlastung" wird zum individuellen Problem der Beschäftigten.

Im neuen Steuerungsmodus kommt es $\mathrm{zu}$ einem Paradigmenwechsel von einer aufwands- $\mathrm{zu}$ einer ertragsorientierten Steuerung. In der aufwandsorientierten Steuerung ist das Ergebnis (produzierte Menge, erbrachte Dienstleistungen, Umsatz, Ertrag) eine abhängige Variable des Aufwands. Ausgangspunkt jeder Planung, jeder Steuerung und natürlich auch jeder Rationalisierung ist die Prüfung der vorhandenen Ressourcen und die Definition der Beziehung zwischen Ressourcen und Aufwand einerseits und dem gewünschten Ergebnis andererseits. In der ertragsorientierten Steuerung wird dagegen zuerst das Ergebnis definiert, dem dann der Aufwand variabel angepasst werden muss (Peters/ Sauer 2005; Ehlscheid et al. 2006; Menz 2009). Dies alleine wäre allerdings weder neu - auch früher haben Unternehmen definiert, wo sie hinwollen und was erreicht werden soll - noch würde sich daraus alleine ein besonderes Rationalisierungs- und Steuerungspotenzial ergeben.

Der erste Unterschied zur „alten“ aufwandsorientierten Steuerung besteht darin, dass nun nicht mehr produktionsökonomische, sondern markt- und kapitalmarktökonomische Überlegungen die Grundlage der Zieldefinition sind. Den Ausgangspunkt bilden jetzt zum einen abstrakte Renditeerwartungen, Marktstrategien („Weltmarktführer“) sowie Wettbewerbskennzahlen (Benchmarks, Kundenzufriedenheits-Indizes etc.); zum anderen dynamische Ziel- und Ergebnisvorgaben, die durch Exploration des Ist-Zustandes entwickelt werden, d.h. das jeweilige Vorjahresergebnis wird zur Grundlage des neuen Jahresziels - plus einer Steigerungsrate: „Wir haben jedes Jahr die freundliche Aufforderung, $10 \%$ Produktivität zu machen", so ein Abteilungsleiter im Interview. In die Ergebnis- und Zieldefinition wird damit ein dynamisches Element eingebaut, weil Stagnation Rückschritt wäre: Die Organisation muss jedes Jahr besser, schneller, billiger werden. Die abstrakten und dynamischen Ziele ertragsorientierter Steuerung markieren den operativen „Fortschritt“.

Hinzu kommt eine neue Spielregel für die Übersetzung abstrakter und dynamischer Zielvorgaben in operative, bearbeitbare Ziele: Output und Ertrag müssen jedes Jahr steigen, aber der Aufwand darf nicht in gleichem Maße wachsen, sondern sollte am besten gleichbleiben oder sogar sinken. Das ist der zweite Unterschied zur „alten“ Aufwandsorientierung. Das Ziel ist ein größerer Output ohne zusätzlichen, allenfalls mit unterproportional gesteigertem Input - die Relation von Aufwand und Ergebnis selbst wird dynamisiert. Das Ergebnis ist eine "systematische Überlastung" der Organisation (Kratzer 2003). Die Organisation ist überlastet, weil sie auf dem jeweils gegebenen Stand das geforderte Ergebnis eigentlich gar nicht schaffen kann. Und sie ist systematisch überlastet, weil ihr ganz gezielt „herausfordernde“ oder eben „unerreichbare" Vorgaben gemacht werden. Nur so entfalten die Vorgaben, das ist die Theorie dahinter, ihr Motivationspotenzial und den „notwendigen“ Druck.

Der dritte Unterschied besteht in einem veränderten Zeitbezug der Planung und Steuerung. In einer aufwandsorientierten Steuerung ist die Zeitmenge eine der wichtigsten Planungsgrößen und die Minimierung des Zeitbedarfs ein zentrales Rationalisierungsziel. Auch im neuen Steuerungsmodus bleibt die Zeit das wichtigste Medium der Vermittlung abstrakter und konkreter Vorgaben, aber nun geht es nicht mehr in erster Linie um die Zeitmenge, sondern um den Zeitpunkt (Kratzer/Sauer 2007): Gesteuert wird wesentlich über die Festlegung der Termine, über zeitliche Ergebnis- und Zielvorgaben; der Aufwand, also die Zeitmenge, kommt dann erst in zweiter Linie ins Spiel, eben als von den operativen Einheiten und/oder den Beschäftigten selbst anzupassender Faktor.

Die Unternehmen geben aber den Steuerungs- und Rationalisierungsprozess nur teilweise aus der Hand: Zum einen wird natürlich auch weiter klassisch rationalisiert. Deswegen trifft man in den Unternehmen sowohl auf selbstgesteuerte Arbeitsprozesse und „ermächtigte“ Subjekte wie auch - gleichzeitig - auf Standardisierungsprozesse, Personalabbau usw. Standardisierung, Selbststeuerung und eine „neue Ökonomie der Unsicherheit“ (Marrs 2008; Kämpf 2008) sind nicht, wie es oft dargestellt wird, ganz unterschiedliche oder alternative Rationalisierungsstrategien, sondern Bestandteile eines (allerdings differenzierten) Steuerungsmodus (Kratzer et al. 2008). Zum anderen aber wird der Top-down-Prozess des „Herunterbrechens“ von Ergebnisvorgaben und Zielen auf (und durch) die Ebenen der Organisation flankiert durch den Bottom-up-Prozess eines immer aufwändigeren Controllings, das der ständigen Rückkoppelung des erreichten Standes „von unten nach oben“ dient. So holen sich die Unternehmen ein beträchtliches Stück Kontrolle über den Rationalisierungsprozess wieder von den Subjekten zurück. Deshalb treten neue Steuerungsformen nicht nur als reine Ertragsorientierung („Tut, was ihr wollt, aber seid profitabel“), sondern stets im Verbund mit immer intensiveren (und extensiveren) Controlling- und Reportingverfahren und -systemen auf. Es handelt sich bei der Ertragsorientierung daher immer um gesteuerte und vor allem auch kontrollierte Ertragsorientierung.

\section{Steuerungslücke": Wie der Leistungsdruck ankommt}

Auf jeder Ebene der Organisation muss ein Transformationsprozess stattfinden, in dem abstrakte Vorgaben in konkrete, bearbeitbare Anforderungen verwandelt werden. Auf diese Weise werden die Vorgaben - wie in einer "Zielkaskade“ - von oben nach unten "heruntergebrochen“. Auf der untersten Führungsebene bzw. auf der Abeitsebene treffen nun endgültig und unhintergehbar abstrakte Vorgaben auf konkrete Gegebenheiten, hier muss ein abstraktes, auf „motivierende Überforderung“ setzendes Steuerungsprinzip in konkrete Arbeitsleistung transformiert werden. Dabei wird die Arbeitsebene, die Welt des Konkreten, zu einer Grenze ergebnisorientierter Steuerung: Je unrealistischer die Kennzahlen, desto weniger Einfluss haben sie auf den tatsächlichen Arbeitsablauf. Unsere Befunde zeigen, dass die Ziel- und Ergebnisvorgaben einen unhintergehbaren Rahmen der Leistungsverausgabung schaffen, dass sie aber keinesfalls bruch- oder widerspruchslos einfach übernommen werden (Nickel et al. 2008, S. 117ff.).

Es sind nicht die Ziel- und Ergebnisvorgaben, die die Beschäftigten „antreiben “ - jedenfalls nicht direkt; und auch der Stellenwert von Führung oder, andersherum, der Einfluss der Führung auf die Leistungsverausgabung scheint gering. Zum einen können die Führungskräfte die alltägliche Arbeit der Beschäftigten oft gar nicht überblicken, zum anderen sind den Führungskräften in ganz entscheidenden Fra- 
gen selbst die Hände gebunden: Natürlich kontrollieren sie das Ergebnis, aber sie können weder Vorgaben noch Ressourcen wirklich beeinflussen. Vor diesem Hintergrund bezeichnen wir mit dem Begriff „Steuerungslücke“, dass die konkreten Gegebenheiten zur Grenze zielorientierter Führung werden. „Steuerungslücke“ heißt aber nicht, dass nicht gesteuert würde. Steuerung funktioniert hier hauptsächlich über zwei Mechanismen:

(1) Indirekte Steuerung durch Rahmenbedingungen: Gesteuert werden im Wesentlichen die Rahmenbedingungen. $\mathrm{Zu}$ diesen gehört erstens der Arbeits- und Zeitdruck selbst, der dazu zwingt, mehr und vielfältiger werdende Arbeit in der gleichen (oder weniger) Zeit zu schaffen. Zweitens kommt hier auch der Kunde ganz zentral ins Spiel: "Leistung" wird nicht nur an das Unternehmen verkauft, sondern auch mehr oder weniger direkt an die Kunden. Das dritte Element sind die vielfältigen Prozess- und Systemzwänge, in die die Beschäftigten eingebunden sind. Angestelltenarbeit ist oft Einzelarbeit, diese ist aber hochgradig involviert und vernetzt. Mehr denn je sind die Angestellten Teil eines Prozesses und ihre Tätigkeit ist vor allem eine Schnittstelle, an der jeweils vor- und nachgelagerte sowie parallele Prozesse zusammenlaufen. Dadurch multipliziert sich die Zahl der Ansprechpartner und „Kunden“. Man hat viele „Auftraggeber“ und ebenso viele Abnehmer für die eigene Leistung.

(2) Vertrauen auf die Eigenmotivation: Unternehmen und Führungskräfte setzen in ganz basaler und auch traditioneller Weise auf die Eigenmotivation der Angestellten und können das auch: Alle Befragten bezeichnen sich als vor allem intrinsisch motiviert. Allen ist in erster Linie wichtig, ihre Arbeit gut zu machen und mit eigenem Interesse dabei zu sein. Im Gegenzug wird eine instrumentelle Arbeitsauffassung abgelehnt und kommt nur als eine Not(wehr)Strategie infrage, die sich zudem kaum durchhalten lässt: Dienst nach Vorschrift funktioniert nicht und verstößt gegen den eigenen Anspruch an die Arbeit (Nickel et al. 2008)

Ingesamt war deutlich, dass sich die Befragten ganz explizit einer ,instrumentellen" Logik entziehen oder sogar widersetzen. Die Frage, woher ihre Leistungsbereitschaft und Motivation kommt, beantwor- ten alle intrinsisch: Man will einfach gute Arbeit abliefern, freut sich am positiven Feedback durch Kunden, ist von der Technik fasziniert usw. Solche Antworten spiegeln natürlich auch ein Stück weit „soziale Erwünschtheit" wieder, eine Selbstbeschreibung, die sich im traditionellen Referenzrahmen intrinsisch motivierter Angestelltenarbeit bewegt und wohl auch bewegen muss. Der Verdacht liegt aber nahe, dass diese Antworten auch einen gewissen Widerstand gegen die instrumentelle Logik erfolgs- bzw. ertragsorientierter Steuerungsformen zum Ausdruck bringen: Die Angestellten in unserer Untersuchung (und zwar alle Gruppen) empfinden die instrumentelle Logik neuer Steuerungsformen nicht nur nicht als adäquaten Bezugsrahmen, sondern sogar als Angriff auf ihr Selbstverständnis als jemand, den man eben nicht per Vorgaben und Controlling zum Erfolg treiben muss. Das Spektrum der Umgangsweisen reicht dabei von „distanzierter Hinnahme“ bis zur „eigensinnigen Umformung".

Bei der distanzierten Hinnahme wird zwar anerkannt, dass es diese Vorgaben, Kennzahlen usw. gibt und man auch danach bewertet wird; als Handlungsorientierung werden sie aber explizit abgelehnt. Der Ausgangspunkt für die Beschäftigten ist hier der Bruch zwischen strategischer und operativer Ebene: Weil beide Ebenen systematisch entkoppelt sind, weil eben auf der Strategieebene gerade nicht danach gefragt wird, was operativ "machbar“ ist, verbannen sie die Vorgaben und Kennzahlen in eine Parallelwelt von Handlungslogiken und Steuerungsprinzipien, von der sie zwar belästigt werden, die aber im Grunde nichts mit ihnen und ihrer Arbeit zu tun hat.

„Wenn Sie jetzt sagen: Ich war heute besonders lieferfähig, deswegen habe ich einen tollen Tag gehabt, dann wäre ich jeden Tag frustriert ... (allgemeines Lachen). Also von daher kann das nicht die Messgröße sein, an der wir unsere Arbeit messen. Persönlich für uns" (Gruppendiskussion Auftragsbearbeitung).

Bei der eigensinnigen Umformung werden die neuen Steuerungslogiken in das professionelle Selbstverständnis integriert, dabei aber auch - eigensinnig - umgeformt. Die eigene Orientierung ist ganz eindeutig auch darauf ausgerichtet, durch Leistung und Professionalität einen Beitrag zur ökonomischen Entwicklung des Betriebs zu leisten. Aber die Beschäftigten drehen das Verhältnis von Leistung und Er- trag eben wieder um: Nur wenn der Kunde tatsächlich zufrieden bzw. das Produkt oder die Dienstleistung wirklich gut ist, dann hat man ökonomischen Erfolg.

„... ich muss ja zum Schluss den Erfolg haben und den Erfolg bestätigt mir ja der Kunde. Wenn der Kunde zufrieden ist mit dem, was ich geleistet habe, dann ist er happy. Somit ist meine Company happy“ (Techniker).

Der Kunde und dessen Qualitätsorientierung werden so zum Verbündeten bei der Aufrechterhaltung fachlich-inhaltlicher Orientierungen und er wird auch gegen die Rationalisierungs- und Ökonomisierungsansätze des eigenen Unternehmens in Stellung gebracht.

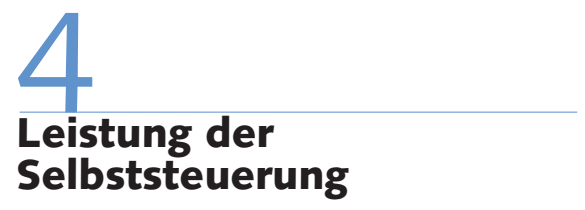

$\mathrm{Ob}$ in der Formulierung eines „Rationalisierungsdilemmas" (Berger/Offe 1984), „verantwortlicher Autonomie“ (Friedman 1987) oder des ,informellen Vertrags“ (Kotthoff 1998): Dass Angestellte ihre Leistungsverausgabung teilweise oder weitgehend selbst steuern, ist ein geradezu konstitutives Merkmal von Angestelltenarbeit. Im neuen Steuerungsmodus bekommt die Selbststeuerung der Leistungsverausgabung aber eine zweite Ebene. Neben die Selbststeuerung der eigenen Leistungsverausgabung tritt - mit zunehmender Wichtigkeit - eine neue Leistung: die Leistung der Selbststeuerung. Diese besteht allgemein gesprochen darin, immer wieder aufs Neue selbst die Voraussetzungen für eine zielgerichtete Leistungsverausgabung herzustellen. Die Leistung der Selbststeuerung ist die Leistung der Bewältigung des Problems systematischer Überlastung.

Empirisch wird dies vor allem daran deutlich, dass die Gesamttätigkeit der Beschäftigten in zwei Tätigkeitsbereiche zerfällt: einerseits in den Bereich der ,eigentlichen“ Arbeit, der „Kernarbeit", anderseits in einen Bereich, den man „Organisationsarbeit" nennen könnte. Die Beschreibung der „Kernarbeit“ ist in den Interviews die Antwort auf die Frage, was die Beschäftigten machen: Entwicklung, Einkauf, Reparatur, Auftragsbearbeitung usw. Die „Organisationsarbeit" ist die Antwort auf die Frage, wie ihr Arbeitstag aussieht: Abstimmung mit anderen Abteilungen, Kollegen 
oder Kunden, die Beantwortung von Anfragen, die Beteiligung an Standardisierungsprojekten, die Erfüllung von Reporting- und Controlling-Anforderungen, Lösung kleinerer oder größerer Probleme (das System funktioniert nicht, die Preisliste stimmt nicht, die Spedition liefert nicht ...) usw. Die Anteile beider Tätigkeitsbestandteile variieren zwar zwischen den verschiedenen Angestelltengruppen, aber nahezu alle beschreiben, dass ihre Tätigkeit in zunehmendem Maße aus mehr als der Kernarbeit besteht, und nahezu alle beschreiben diese Entwicklung als problematisch. Das größte Problem ist dabei, dass die Organisationsarbeit in der Regel zunehmende und vor allem ungeplante Zeitanteile bindet und so wesentlich zur Überlastung beiträgt. Die Organisationsarbeit ist auch schwer greifbare Arbeit: Sie ist genau der Grund, warum Beschäftigte sagen: "Soviel gemacht und nichts geschafft".

Weil die Organisation ihr organisationelles Problem der systematischen Überlastung nur noch teilweise selber löst, müssen die Beschäftigten in wachsendem Umfang „Organisationsarbeit" leisten. Die Zunahme der Organisationsarbeit ist die Folge der Transformation des organisationellen in ein individuelles Problem - und sorgt so für die Überlastung der Beschäftigten und dafür, dass Selbststeuerung nun nicht mehr nur auf die Arbeitsausführung, sondern eben auch auf die Bewältigung organisationeller Probleme ausgerichtet ist.

Die Leistung der Selbststeuerung - und damit auch die Arbeit, die mit ihr verbunden ist, also die Organisationsarbeit - ist, anders als die „alte“ Selbststeuerung der Leistung, allerdings „unsichtbare“ Leistung, weil sie selbst kein Ergebnis hat. Ihr Ergebnis ist, dass es überhaupt ein Arbeitsergebnis gibt bzw. geben kann. Sie wird wenn überhaupt - nur negativ, als mangelnde oder mangelhafte Leistung sichtbar: Wenn es eben kein Arbeitsergebnis gibt oder das Ergebnis nicht den Anforderungen entspricht, wenn Termine nicht eingehalten werden, Kunden unzufrieden sind usw. Die Folge sind Anerkennungsdefizite, die umso größer sind, je weniger sie über die Anerkennung des Arbeitsergebnisses der Kernarbeit aufgefangen werden. Während die Entwickler vor allem die ungeplanten Zeiterfordernisse der „Organisationsarbeit" kritisierten, empfanden die produktionsnahen Angestellten, die in relativ standardisierbare Arbeitsprozesse ein- gebunden sind (also etwa Auftragsbearbeitung) die „Unsichtbarkeit“ der Leistung der Selbststeuerung als zentrales Problem, weil die Kernarbeit „von Kopfarbeit zum Knöpfchendrücken" mutiert, die wachsende Bedeutung der Organisationsarbeit und der Leistung der Selbststeuerung aber keine Anerkennung findet.

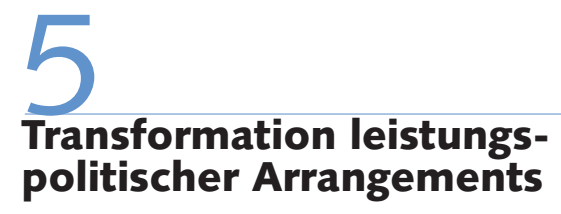

Leistungssteuerung erfolgt bei Angestellten nicht über ein einzelnes Instrument oder einen einzelnen „Mechanismus“ (Marrs 2008), sondern über - unterschiedlich ausgeprägte - leistungspolitische Arrangements. Im Begriff des „Arrangements" kommt zweierlei zum Ausdruck: zum einen, dass das Zustandekommen konkreter Leistung immer das Ergebnis nicht nur verschiedener, sondern auch unterschiedlicher Interessen ist, die sich eben „arrangieren“ müssen. Zum anderen verweist der Begriff darauf, dass Leistungssteuerung nur über das Zusammenwirken, das „Arrangement“, mehrerer Instrumente und Mechanismen funktioniert.

In klassischen leistungspolitischen Arrangements wie der „verantwortlichen $\mathrm{Au}$ tonomie" (Friedman 1987) oder dem impliziten bzw. „informellen“ Vertrag (Kotthoff 1998) bringen Beschäftigte eine hohe Leistungsbereitschaft, die Übernahme von Mitverantwortung für das Unternehmen und ihre Loyalität zum Unternehmen ein. Das Unternehmen gewährt dafür recht weitgehende Freiräume in der Arbeitsausführung und vergleichsweise privilegierte Arbeitsbedingungen (vor allem: Beschäftigungssicherheit, relativ hohe Einkommen, Entwicklungs- bzw. Karriereperspektiven). Dass dieses spezifische leistungspolitische Arrangement unter Druck ist, ist schon länger klar: Abnehmende Beschäftigungssicherheit, eingeschränkte Karriere- und Entwicklungsperspektiven, Erosion der betrieblichen Sozialordnung, zunehmend begrenzte Freiräume durch Standardisierungsprozesse, Überformung intrinsischer, fachlich-inhaltlicher Interessen durch ökonomische Verwertungskalküle lauten die Diagnosen (Kotthoff 1998; Kratzer 2003; Boes/Trinks 2006; Grewer et al. 2007; Vester et al. 2007; Nickel et al. 2008; Kämpf 2008; Kotthoff/Wagner 2008; Marrs 2008).
Zu beobachten ist zum Teil die Erosion, vor allem aber die Transformation leistungspolitischer Arrangements: Ganz eindeutig steht die „verantwortliche Autonomie" der Angestellten zunehmend unter Markt- und Verwertungsdruck und ganz eindeutig spielen dabei ertragsorientierte Vorgaben und Kennzahlen eine zentrale Rolle. Auf der anderen Seite kommt es aber weder flächendeckend zu einer völligen Erosion der konstitutiven Merkmale verantwortlicher Autonomie noch zu einer „einfachen“, sozusagen distanzlosen, Umformung fachlich-inhaltlicher Interessen in eine individuelle Ertragsorientierung (Kotthoff/Wagner 2008). Und auch die von den Unternehmen durchaus angestrebte - Durchsteuerung nach den neuen Prinzipien funktioniert nur begrenzt: Nach wie vor bildet die Arbeitsebene der Angestellten eine Grenze nicht nur tayloristischer, sondern auch marktökonomischer Durchsteuerung.

Es sieht eher danach aus, dass die neuen leistungspolitischen Arrangements auf widersprüchliche Weise das "Alte“ mit dem „Neuen“ zu einem einigermaßen funktionalen Ganzen vereinen: Die instrumentelle Logik der Erfüllung abstrakter und dynamischer Ziel- und Ergebnisvorgaben tritt neben intrinsische Interessen und Motive, die Leistung der Selbststeuerung neben die Selbststeuerung der Leistung usw. Die „Steuerungslücke“ macht die Sache aber keineswegs gemütlicher, da die Beschäftigten nun sozusagen "ungesteuert“ mit systematischer Überlastung konfrontiert werden und neben ihrem Arbeitsproblem auch noch das organisationelle Problem der Bewältigung systematischer Überlastung zu lösen haben - und das ohne adäquate Anerkennungsverhältnisse und bei klar verschlechterten Tauschrelationen.

Die neue „Steuerungslücke“ der Angestelltenarbeit ist daher weder einfach der verlängerte Arm eines neuen Steuerungsmodus noch dessen absolute Grenze. Sie ist vielmehr eine einigermaßen funktionierende Synthese aus der neuen Logik ertragsorientierter Steuerung und der ,alten “ Logik leistungspolitischer Arrangements bei Angestellten. Der neue Steuerungsmodus funktioniert nicht trotz, sondern wegen seiner Widersprüche, nicht trotz, sondern wegen seiner Bezugnahme auf den „alten“ Modus der „verantwortlichen Autonomie“.

Allerdings ist die „Steuerungslücke“ gerade kein stabiles Arrangement. Die „Steue- 
rungslücke“ ist eben eine „Lücke“ und deshalb instabil: Schließungs- und Öffnungstendenzen oszillieren um den Widerspruch der abstrakten (Durch-)Steuerung konkreter, zumal „geistiger" Arbeit. Mal schnüren Kennzahlen, Controlling, Standardisierung und Prozesszwänge die Freiräume ein, mal werden diese erweitert, wenn die Spielräume für die Leistung der Selbststeuerung zu klein werden und nicht nur Demotivation und Distanzierung drohen, sondern auch die Gefahr besteht, dass das Problem der systematischen Überlastung wieder auf die Organisation selbst zurückfällt.

\section{Leistungspolitische Impulse des ERA}

Im Idealfall ist die „Steuerungslücke“ die Lösung des unternehmerischen Steuerungsproblems und geschätzter Freiraum der Beschäftigten, im Normalfall ist sie das zentrale leistungspolitische Konflikt- und Gestaltungsfeld. Und hier kommen die Leistungsentgelte als mögliches Gestaltungs- und Regulierungsinstrument ins Spiel: Leistungsentgelte könnten - so ja nicht zuletzt eine der Hoffnungen, die die IG Metall mit den ERA-Tarifverträgen verbunden hat - ein Baustein einer neuen Leistungspolitik bei Angestellten sein. Allerdings zeigen unsere Befunde, dass Leistungsentgelte für die Leistungssteuerung im Angestelltenbereich bislang praktisch keine Rolle gespielt haben und dass auch die ERA-Einführung daran zunächst nicht viel geändert hat.

Nur in wenigen Fällen kam es zeitgleich mit der Umgruppierung nach dem ERA auch zu einer Neugestaltung der Leistungsentgelte, in der Regel wird der Prozess der Eingruppierung zeitlich von der Einführung neuer Leistungsentgeltstrukturen entkoppelt (Schulte 2008, S. 168ff.). Dies hat vor allem ganz pragmatische Gründe: Die Komplexität des ERA und der Aufwand einer kompletten Neueingruppierung führen häufig dazu, dass schlichtweg nicht genügend "Luft“ zu sein scheint, um sich auch noch mit Fragen der Leistungsentgeltgestaltung auseinanderzusetzen. ${ }^{2}$ Auf den ersten Blick passiert bislang also noch nicht viel. Auf den zweiten Blick wird aber deutlich, dass mit der ERA-Einführung dennoch Impulse verbunden sind, die dafür sprechen, dass wir nicht das (frühzei- tige) Ende einer leistungspolitischen Gestaltungsoption beobachten, sondern möglicherweise den Anfang einer intensiveren Auseinandersetzung mit leistungspolitischen Fragen im Angestelltenbereich.

Ein wichtiger Impuls kommt von den Angestellten selbst: Gegenüber der bisherigen Praxis und entgegen dem weitverbreiteten Vorurteil, Angestellte stünden Leistungsentgelt prinzipiell kritisch gegenüber, wird eine Ausweitung leistungsbezogener Entgeltbestandteile von den von uns befragten Angestellten befürwortet, teils sogar explizit eingefordert. Fragt man nach den Gründen, dann wird schnell deutlich, dass es den Befragten vor allem um eine "bessere" oder auch "gerechtere" Anerkennung von Leistung geht. Die Bedeutung, die Beschäftigte der Anerkennungsdimension von Leistungsentgelten zumessen, hängt einerseits eng mit den Befunden zur Leistung der Selbststeuerung zusammen, die eine systematische Anerkennungslücke erzeugt. Von einer „echten“ und real praktizierten Leistungsbewertung erwarten die Befragten, dass auch die bislang systematisch unterbelichteten Aspekte ihrer alltäglichen Arbeitsleistung berücksichtigt werden. Die Anerkennung der Selbststeuerung als Arbeitsleistung ist damit auch ein wichtiger Schritt zur Explizierung der Arbeitsanforderungen und dies wiederum ist eine grundlegende Voraussetzung ihrer Begrenzung. Zum anderen kommt andererseits auch der Frust über die ERA-Einführung zum Ausdruck: Einige Angestelltengruppen, in unserem Sample vor allem die Teamassistentinnen und die produktionsnahen Angestellten, sahen sich durch die ERAEingruppierung massiv abgewertet - und das heißt nicht zuletzt auch: in ihrer Leistung, oft auch „Lebensleistung“ nicht anerkannt. ${ }^{3}$ Das Verhältnis von Leistung und Gegenleistung stimmt angesichts schon lange wachsender Anforderungen nicht wirklich - und erst recht nicht angesichts einer Neubewertung des Arbeitsplatzes nach dem ERA.

Ein weiterer wichtiger leistungspolitischer Impuls, der mit der ERA-Einführung verbunden ist, resultiert daraus, dass sich im Zuge der Optionen der Leistungsentgeltgestaltung, die ERA bietet, alle Beteiligten, insbesondere aber natürlich auch Betriebsräte, nun zumindest mit dem Thema „Leistungsentgelt im Angestelltenbereich“ auseinandersetzen müssen. So wurden in einigen Betrieben auch schriftliche Vereinbarungen über eine spätere Neugestaltung der Leistungsentgelte getroffen. Neben den - hinlänglich bekannten - Leistungsbeurteilungen (Menz 2009; Menz et al. 2008) stehen für den Angestelltenbereich nun auch die Methoden Kennzahlenvergleich und Zielvereinbarungen zur Debatte. Keine Methode scheint allerdings den spezifischen Herausforderungen bezüglich kollektiver Regulierbarkeit, der Möglichkeiten leistungspolitischer Einflussnahme und der Berücksichtigung der Interessen der Beschäftigten gerecht zu werden.

Bislang hatten die Tarifbeschäftigten praktisch ausschließlich mit Leistungsbeurteilungen zu tun. Als Feedback-Instrument erfüllt die Leistungsbeurteilung durch die Berücksichtigung „weicher" Faktoren und die Bewertung des Leistungsverhaltens zwar prinzipiell einige der Kriterien einer ganzheitlicheren Anerkennung der Arbeitsleistung, wird aber in der betrieblichen Praxis von niemandem als leistungspolitisches Instrument wahr- und ernstgenommen. Die meisten Beschäftigten konnten uns nicht sagen, wie hoch der prozentuale Anteil ihrer leistungsbezogenen Vergütung war - und es hat sie auch nicht sonderlich interessiert, weil dieser Anteil kaum variiert, und wenn, dann nach Kriterien, die wenig bis gar nichts mit ihrer Leistung zu tun haben. Immer wieder wurde die bekannte Kritik an der „subjektiven Bewertung“ von Vorgesetzten geäußert, die mehr mit „Nasenfaktor", Willkür oder Seniorität denn mit Leistung zu tun hat (Menz 2009).

Die von den meisten Betriebsräten favorisieren Prämienentgelte auf Kennzahlenbasis haben zwar den Vorteil, dass individuelle Probleme wieder stärker auf einer kollektiven Ebene regulierbar werden, treffen bei den meisten Angestellten aber kaum auf Gegenliebe: Deren Leistung, so wird es immer wieder in den Interviews deutlich, sei nicht zähl- und messbar und gerade die indirekten Beiträge der „Leistung der Selbststeuerung " fallen dann wohl (wieder) unter den Tisch.

Bleiben die Zielvereinbarungen: Die Erfahrungen sind hier bescheiden und auch unsere Empirie blieb im Hinblick auf Zielvereinbarungen auf Interviews mit

2 Ganz ähnlich die Befunde der ERA-Begleitforschung aus den Tarifbezirken Baden-Württemberg (Bahnmüller/Schmidt 2009), Niedersachen (Kuhlmann/Sperling 2009) und Thüringen (Schmierl 2009).

3 Vgl. dazu auch die in Fußnote 2 genannten Studien. 
außertariflich Beschäftigten (überwiegend: Ingenieure) beschränkt. Es kommen vor allem die - zum Teil bereits bekannten - kritischen Argumente (keine "echte“ Verhandlungssituation, Einengung der Perspektive auf wenige leistungsrelevante Ziele, Beitrag zur Leistungsdynamisierung). Zielvereinbarungen waren allerdings zugleich das einzige Instrument, das, wenn auch nur von wenigen, überhaupt mit leistungspolitischen Fragen in Beziehung gesetzt wurde: Nur im Rahmen dieser Methode, so die positiv gestimmten Befragten, könne überhaupt eine Auseinandersetzung über die Rahmenbedingungen der Leistungsverausgabung stattfinden, also über verfügbare Ressourcen, ungeplante Änderungen etc.

\section{7 \\ Leistungsentgelte im neuen leistungspolitischen Arrangement}

Für einen Teil der Angestellten, das zeigt unsere Untersuchung ganz deutlich, geht es bei der ERA-Einführung längst nicht nur um eine Entgeltreform: Zur Debatte stehen vielmehr konstitutive Merkmale der leistungspolitischen Arrangements und die Abwertung von Arbeit und (Lebens)Leistung. Beides hat seine weiteren Ursachen aber auch in einem neuen Steuerungsmodus, der sowohl die Mehrdimensionalität leistungspolitischer Arrangements wie auch den Arbeitskraftbezug von Leistung systematisch ignoriert. Es geht - natürlich - um Entgelt, aber eben nicht nur. Leistungspolitik bei Angestellten ist deshalb auch nicht Sache eines Instruments, son- dern muss mehrdimensional sein; sie bedeutet immer: Gestaltung leistungspolitischer Arrangements in ihren verschiedenen Dimensionen.

Leistungsentgelte sind deshalb nicht die Lösung, können jedoch ein wichtiger Baustein sein. Leistungsentgelte sind eine institutionelle Gelegenheitsstruktur, eine Chance, die Auseinandersetzung darüber, was Leistung überhaupt ist, welchen Wert sie hat und in welchem Verhältnis sie zu den anderen Dimensionen leistungspolitischer Arrangements steht, mit den Akteuren selbst und nachhaltig zu führen. Die ERAEinführung beinhaltet deshalb in zweifacher Hinsicht leistungspolitische Impulse: Mit ihr rückt nicht nur das Thema Leistungsentgelt auf die Agenda, sondern auch das Thema „Leistung“.

Dass beim Thema Leistungsentgelt im Angestelltenbereich - noch - nicht viel passiert ist, liegt zum einen an fehlenden Kapazitäten und häufig auch fehlenden Kompetenzen, aber auch an erheblichen Vorbehalten. Leistungsentgelte, so ein erster Kritikpunkt, sind vor allem Instrumente der Leistungsdynamisierung, nicht der Begrenzung oder Regulierung von Leistungsdruck. Zumindest bislang und zumindest in den von uns untersuchten Betrieben und Angestelltengruppen spielen Leistungsentgelte als Instrumente der Leistungssteuerung praktisch keine Rolle. Der Leistungsdruck kommt dagegen aus der systematischen Überlastung und ihrer Transformation in ein individuelles Problem. Leistungsentgelte verkörpern, so der zweite Kritikpunkt, geradezu das „Leistungsprinzip“, also das hochproblematische Prinzip, dass Entgelt, Sicherheit, gesellschaftliche Teilhabe etc. sich nur an der (ökonomischen) Leistung bemessen (sollen). Aber mit der Durchsetzung des neuen „Marktprinzips“ (Menz 2009) erhält auch das Leistungsprinzip einen anderen Stellenwert: Weil das „Leistungsprinzip“ - sozusagen: immerhin auf die individuelle Leistung rekurriert und damit noch ein arbeitskraftabhängiges Maß der Leistung hat, kann es gerade ein Gegengewicht zum Marktprinzip und seiner arbeitskraftunabhängigen Bewertung von „Leistung“ am (Markt-)Erfolg sein.

Der Blaupause der industriellen Leistungsentlohnung sind dabei allerdings enge Grenzen gesetzt: Unsere Befunde zur „Steuerungslücke“ und zur „Leistung der Selbststeuerung" machen zweierlei deutlich: Erstens übernehmen die Beschäftigten nicht „einfach“ die abstrakten und dynamischen Vorgaben ergebnisorientierter Steuerungssysteme und internalisieren sie als eigene Handlungsorientierungen. Die Ziel- und Ergebnisvorgaben sind nur eine von mehreren, oft widersprüchlichen Anforderungen und Bedingungen, mit denen sie sich arrangieren müssen. Das erzeugt viel Druck und neuartige Beanspruchungen, ist aber nach wie vor auch ein Freiraum, Teil einer Selbstständigkeit in der Arbeitsausführung, die man ihnen nicht so einfach nehmen kann - und die sie sich auch nicht nehmen lassen wollen. Zweitens erwachsen daraus spezifische Herausforderungen für eine neue Leistungspolitik im Angestelltenbereich, die eine schlichte Übertragung des Erfolgsmodells industrieller Leistungsregulierung verhindern: Wo Leistung nicht (mehr) direkt, sondern vor allem indirekt gesteuert wird, fällt auch eine direkte Regulierung der Leistungsanforderungen und -bedingungen schwer. 


\section{LITERATUR}

Bahnmüller, R. (2001): Stabilität und Wandel in der Leistungsentlohnung, in: WSI-Mitteilungen 7, S. 426-433

Bahnmüller, R./Schmidt, W. (2009): Riskante Modernisierung des Tarifsystems. Die Reform der Entgeltrahmenabkommen am Beispiel der Metall- und Elektroindustrie Baden-Württembergs, Berlin

Berger, U./Offe, C. (1984): Das Rationalisierungsdilemma der Angestelltenarbeit, in: Offe, C. (Hrsg.): „Arbeitsgesellschaft “ - Strukturprobleme und Zukunftsperspektiven, Frankfurt/New York, S. 271-290

Boes, A./Trinks, K. (2006): Theoretisch bin ich frei! Interessenhandeln und Mitbestimmung in der IT-Industrie, Berlin

Ehlscheid, C./Tondorf, K. (2006): Zielvereinbarungen zwischen Managementmethode und Entgeltgrundsatz, in: Brunkhorst, C./Burkhard, O./ Scherbaum, M. (Hrsg.): Eine neue AERA. Tarifverträge für die Zukunft, Hamburg, S. 100-119

Ehlscheid, C./Meine, H./Vogt, W. (2006): „Wie viel Geld für wie viel Leistung?" Entlohnungsgrundsätze und Leistungsbedingungen, in: Ehlscheid, C./Meine, H./Ohl, K. (Hrsg.): Handbuch Arbeit - Leistung Entgelt. Tarifanwendungen im Betrieb, Frankfurt, S. 202-317

Friedman, A. (1987): Managementstrategien und Technologie: Auf dem Weg zu einer komplexen Theorie des Arbeitsprozesses, in: Hildebrand, E./ Seltz, R. (Hrsg.): Managementstrategien und Kontrolle. Eine Einführung in die Labour Process Debate, Berlin, S. 99-131

Grewer, H./Matthäi, I./Reindl, J. (2007): Der innovative Ältere. Warum die Entwickleruhr länger als sieben Jahre tickt, München und Mering Huber, B./Schild, A. (2004): Die neuen Entgeltrahmentarifverträge, in: WSI-Mitteilungen 2, S. 102-105

Kämpf, T. (2008): Die neue Unsicherheit. Folgen der Globalisierung für hochqualifizierte Arbeitnehmer, Frankfurt/New York

Kotthoff, H. (1998): Führungskräfte im Wandel der Firmenkultur - QuasiUnternehmer oder Arbeitnehmer?, Berlin

Kotthoff, H./Wagner, A. (2008): Die Leistungsträger. Führungskräfte im Wandel der Firmenkultur - eine Follow-up-Studie, Berlin Kratzer, N. (2003): Arbeitskraft in Entgrenzung - Grenzenlose Anforderungen, erweiterte Spielräume, begrenzte Ressourcen, Berlin Kratzer, N. (2006): Zielvereinbarungen als Instrument der Leistungspolitik, in: Huber, B./Burkhard, O./Wagner, H. (Hrsg.): Perspektiven der Tarifpolitik - Im Spannungsfeld von Fläche und Betrieb, Hamburg, S. $146-159$

Kratzer, N./Menz, W./Nies, S./Sauer, D. (2008): Leistungspolitik als Feld „umkämpfter Arbeit“, in: Prokla 150, S. 11-26
Kratzer, N./Nies, S. (2009): Neue Leistungspolitik bei Angestellten. ERA, Leistungssteuerung, Leistungsentgelt, Berlin

Kratzer, N./Sauer, D. (2007): Welche Arbeitszeitpolitik? Ein neues Verhältnis von Zeitökonomie und Zeitpolitik, in: WSI-Mitteilungen 4, S. $174-180$

Kuhlmann, M./Sperling, H. (2009): Der Niedersachsen-Weg - Tarifregelungen, Einführungsprozesse und Wirkungen des ERA, in: WSI-Mitteilungen 3, S. 127-135

Kurdelbusch, A. (2002): Variable Vergütung in deutschen Großunternehmen - Entgeltsysteme zwischen Flexibilisierung und Flächentarifvertrag. Dissertation an der Ruhr-Universität Bochum, Manuskript

Marrs, K. (2008): Arbeit unter Marktdruck - Die Logik der ökonomischen Steuerung in der Dienstleistungsarbeit, Berlin

Menz, W./Nies, S./Kratzer, N. (2008): Die Interessenpolitik des „nutzbringenden Individuums “. Interessenhandeln und Interessenvertretung in individualisierter Leistungspolitik, in: Arbeits- und Industriesoziologische Studien, Online-Journal der Sektion Arbeits- und Industriesoziologie in der Deutschen Gesellschaft für Soziologie (DGS) 2, S. 27-43

Menz, W. (2009): Die Legitimität des Marktregimes. Leistungs- und Gerechtigkeitsorientierungen in neuen Formen betrieblicher Leistungspolitik, Wiesbaden

Nickel, H./Hüning, H./Frey, M. (2008): Subjektivierung, Verunsicherung, Eigensinn. Auf der Suche nach Gestaltungspotenzialen für eine neue Arbeits- und Geschlechterpolitik, Berlin

Peters, K./Sauer, D. (2005): Indirekte Steuerung - eine neue Herrschaftsform. Zur revolutionären Qualität des gegenwärtigen Umbruchprozesses, in: Wagner, H. (Hrsg.): „Rentier' ich mich noch?“ Neue Steuerungskonzepte im Betrieb, Hamburg, S. 23-58

Schmierl, K. (1995): Umbrüche in der Lohn- und Tarifpolitik - Neue Entgeltsysteme bei arbeitskraftzentrierter Rationalisierung in der Metallindustrie, Frankfurt/New York

Schmierl, K. (2009): ERA-Erfahrungen in Thüringen - Befunde aus Pilotbetrieben, in: WSI-Mitteilungen 3, S. 136-142

Schulte, E. (2008): Leistungsregulierung bei der Umsetzung der Entgeltrahmentarifverträge, in: Wagner, H. (Hrsg.): Arbeit und Leistung gestern \& heute. Ein gewerkschaftliches Politikfeld, Hamburg, S. 163-172 Vester, M./Teiwes-Kügler, C./Lange-Vester, A. (2007): Die neuen Arbeitnehmer. Zunehmende Kompetenzen - wachsende Unsicherheit, Hamburg 